\title{
On an Evolution Equation for a Non-Hypoelliptic Linear Partial Differential Operator from Stochastics
}

\author{
K. DopPeL and N. JACOB
}

Neulich hat E. B. DyNkIN [2] einen nichthypoelliptischen linearen partiellen Differentialoperator von gerader Ordnung (mit konstanten-Koeffizienten) eingeführt und untersucht, der aus der Theorie der mehrparametrigen stochastischen Prozesse entstanden ist. Von diesen Betrachtungen von DyNKIN angeregt, haben die Verfasser in der Abhandlung [1] schon ein verallgemeinertes-Dirichlet-Problem für diesen Differentialoperator gelöst. Unser Ziel in der vorliegenden Arbeit ist, das Cauchy-Problem für die entsprechende Evolutionsgleichung (in der Zeitveränderlichen von erster Ordnung) zu untersuchen; ein solches Cauchy-Problem könnte Anwendungen auf Fragen der Stochastik haben.

Недавно Е. Б. Дынкин [2] ввел и исследовал негиперболический линейный дифференциальный оператор четного порядка с постоянными коэффициентами, возникшипся в теории многопараметрических стохастических процессов. Возбуждены рассуждениями E. Б: Дынкин А авторы ранее решили в [1] для әтого оператора обобщенную задачу Дирихле. Цель настоящећ̆ работы - исследовать задачу Коши для соответствующего әволюционного уравнения, первого порядка относительно временноп переменной. Такая задача Коши мог бы иметь применения к вопросам стохастики.

Recently E. B. Dynkis [2] introduced and studied a non-hypoelliptic linear partial differential operator of, even order (with constant coefficients) which originates from the theory of multiparametric stochastic processes. Motivated by the considerations of Dxwkin the authors have solved a generalized Dirichlet problem for this differential operator in their work [1]. Our aim in the present paper is to investigate the Cauchy problem for the corresponding evolution equation (in the time variable of first order); such a Cauchy problem could have applications to some questions from the stochastics.

\section{Introduction}

1.1 We consider in a bounded open set $G \subset \mathbf{R}^{n}$ (which satisfies some conditions, cf. Property $\mathrm{P}$ in 1.2) the non-hypoelliptic linear partial differential operator $L(D)$ of order $2 k(k \in \mathrm{N}, k \leqq n)$ introduced by E. B. DYNkIN [2]; the exact definition of $L(D)$ will be given below in 1.3 . In this paper we want to investigate an initialboundary value problem, which we call the Cauchy problem, for the "abstract" evolution equation

$$
\frac{d}{d t} u(t)+\dot{L} \sim u(t)=f(t)
$$

where $L^{\sim}$ is the closure of the differential operator $L(D)$ in $L^{2}(G), f$ a given function defined on the positive time axis with values in $L^{2}(G)$ and the solutions $u(t)$ are searched among functions defined on the positive time axis with values in a certain subspace of $L^{2}(G)$ which coincides with the domain $D\left(L^{\sim}\right)$ of $L^{\sim}$.

1.2 Let us first recall some notions; notations and results from our earlier paper [1]. Let $G$ be a set in $\mathbf{R}^{n}$ with 
Property P: The bounded open set $G \subset \mathbf{R}^{n}$ is the Cartesian product

$$
G=G_{1} \times \therefore \times G_{k}
$$

of bounded open sets $G_{j} \subset \mathbf{R}^{m_{1}}(1 \leqq j \leqq k), m_{1}+\cdots+m_{k}=n, \mathbf{R}^{n}=\mathbf{R}^{m_{1}} \times \cdots \times \mathbf{R}^{m_{k}}$ with sufficiently smooth boundaries $\partial G_{j}$ (if the boundaries $\partial G_{j}$ are of class $C^{\infty}$ all our considerations surely will be valid).

We write

$$
\partial_{j} G:=G_{1} \times \cdots \times G_{j-1} \times \partial G_{j} \times G_{j+1} \times \cdots \times G_{k} .
$$

By $\mathrm{N}_{0}{ }^{n}$ we denote, as usual, the set of all ordered systems of $n$ nonnegative integers (multi-indices). For $\alpha=\left(\alpha_{1}, \ldots, \alpha_{n}\right) \in \mathbf{N}_{0}{ }^{n}$ and $\beta=\left(\beta_{1}, \ldots, \beta_{n}\right) \in \mathbf{N}_{0}^{n}$ we write $|\alpha|=\alpha_{1}+\cdots+\alpha_{n}$ and $\alpha \leqq \beta$ if $\alpha_{l} \leqq \beta_{i}$ for $1 \leqq l \leqq n$.

With $n, k, m_{j} \in \mathbf{N}$ as in Property $\mathrm{P}$ we put

$$
l_{1}=0, \quad l_{j}:=\sum_{l=1}^{j-1} m_{l} \quad(2 \leqq j \leqq k)
$$

For'each $j(1 \leqq j \leqq k)$ we define $m_{j}$ multi-indices $\varepsilon_{\ell^{\prime}} \in \mathrm{N}_{0}{ }^{n}$ with $\left|\varepsilon_{t_{j}}\right|=1$ each having its only nonvanishing coordinate in the $t_{j}$-th position, $l_{j}+1 \leqq t_{j} \leqq l_{j}+m_{j}$. We introduce the set

$$
\Gamma:=\left\{\dot{\alpha} \in \mathbf{N}_{0}{ }^{n} \mid \alpha=\sum_{j=1}^{k} \varepsilon_{t}\right\} .
$$

Note that $\dot{\Gamma}$ has $m_{1} \ldots m_{k}$ elements. Further, we write

$$
\Gamma^{j}:=\left\{\gamma \in \mathbf{N}_{0}{ }^{n} \mid \gamma=\sum_{\substack{l=1 \\ l \neq j}}^{k} \varepsilon_{t_{l}}\right\} \quad(1 \leqq j \leqq k)
$$

1.3 Now we can introduce the differential operator

$$
L(D):=\sum_{\alpha \in I} D^{2 \alpha}
$$

where we use the abbreviation

$$
D^{\alpha}=D_{1}^{\alpha_{1}} \ldots D_{n}^{\alpha_{n}} \text { with } D_{j}:=-\frac{\dot{ }}{-1} \frac{\dot{\partial}}{\partial x_{j}} .
$$

This operator has also the expression

where

$$
L(D)=\overline{\Delta_{1}} \ldots \Delta_{k}
$$

$$
\Delta_{j}=\sum_{s=1}^{m} D_{l_{j}+s}^{2} \quad(1 \leqq j \leqq k)
$$

denotes the Laplace operator, which acts on functions defined on subsets of $\mathbf{R}^{m},\left(\subset \mathbf{R}^{n}\right)$. As we have shown in [1] the operator $L(D)$ is not hypoelliptic.

1.4 Let $C^{k}(G), k \in \mathbf{N}_{0}$, be the linear space of all complex valued functions $u$ which are $k$ times continuously differentiable in $G$. By $C_{0}{ }^{k}(G)$ we denote the space of all functions $u \in C^{k}(G)$ each having a compact support in $G$. We write also $C_{0}^{\infty}(G)$ $=\bigcap_{k \in N_{0}} C_{0}^{k}(G)$. Further let be

$$
C_{*}{ }^{k}(G):=\left\{u \in C^{k}(G)\left|D^{\alpha} u \in L^{2}(G), \alpha \in \mathbf{N}_{0}{ }^{n},\right| \alpha \mid \leqq k\right\} .
$$



form

In $C_{*}{ }^{k}(G)$ (with $K$ from 1.2) we associate with the operator $L(D)$ the sesquilinear

We put

$$
B(u, v):=\sum_{\alpha \in \Gamma} \int_{G} \overline{D^{\alpha} u(x)} D^{\alpha} v(x) d x .
$$

$$
(u, v)_{r}:=B(u, v)+(u, v)_{0}
$$

where $(.,)_{0}$ denotes the scalar product in $L^{2}(G),(u, v)_{0}=\int_{G^{\prime}} \overline{u(x)} v(x) d x$. Thus, we have a scalar product $(., .)_{\Gamma}$ on $C_{*}{ }^{k}(G)$ with the corresponding norm $\|\cdot\|_{r}$. We denote the completion of $C_{*}{ }^{k}(G)$ with respect to the scalar product $(1.2)$ by $H^{r}(G)$. For the closure of $C_{0}{ }^{\infty}(G)$ in $H^{r}(G)$ we write $H_{0}{ }^{r}(G)$.

On the space $H_{0} \Gamma(G)$ the sesquilinear form (1.1) defines a scalar product equivalent to (1.2), in particular one has the inequalities

$$
B(u, u) \leqq\|u\|_{r^{2}} \leqq c^{*} B(u, u) \text { for all } u \in H_{0} \Gamma(G)
$$

with a constant $c^{*}$ (cf. [1: Lemma 2]). The norm induced by $B\left(.\right.$, . ) on $B_{0} \Gamma(G)$ will be denoted by \|\|$\cdot \mid \|_{r}$.

The elements.of $H_{0} r(G)$ can be interpreted as functions with generalized homogeneous boundary data: Namely, for each $\dot{u} \in H_{0} r(G) \cap C^{k-1}(\bar{G})$ the relation

$$
\left.D^{\beta} u\right|_{\partial, G}=0
$$

holds for all $\beta \in \dot{\mathrm{N}}_{0}{ }^{n}$ with $\beta \leqq \gamma$ for some $\gamma \in \Gamma^{j}(1 \leqq j \leqq k)$ (we proved this assertion in [1: Theorem 4], cf. also [4: p. 28]).

Further for every $u \in H_{0} \Gamma(G)$ the strong $L^{2}$-derivative $D^{r} u$ exists for all $\tau \in N_{0}^{n}$ with $\tau \leqq \sigma$ for some $\sigma \in \Gamma$ (the corresponding assertion for $u \in H^{\Gamma}(G)$ is not valid; cf. [1: Lemma 1], and also [5:'Theorem 1]).

Because the relation (1.4) is valid for functions $u \in H_{0}^{r}(\dot{G}) \cap C^{k-1}(\bar{G})$ one can apply partial integration for functions from the set $X:=H_{0} r(G) \cap C^{\infty}(G)$ and gets

$$
\int_{G} \overline{D^{a} u(x)} D^{a} v(x) d x=\int_{G} \overline{D^{2 a} u(x)} v(x) d x
$$

for all $u ; v \in X$ and all $\alpha \in \Gamma$ (cf. [1: Lemma 5]).

1.5 In the Hilbert space $L^{2}(G)$ we associate, to the differential operator $L(D)$ a linear operator $L$ by

$$
\begin{aligned}
& D(L):=X\left(\subset L^{2}(G)\right), \\
& L u:=L(D) u \text { for all } u \in X
\end{aligned}
$$

This operator is densely defined and has an adjoint operator $L^{*}$, and by. partial integration one sees that $D\left(L^{*}\right) \supset X$ and that $L^{*}$ is also densely defined. The operator $L$ is therefore closable with the closure (smallest closed extension) $L^{\sim}:=L^{* *}$. On the other hand, we have on $X$ a scalar product defined by

$$
\cdot(u, v)_{(k)}:=(L(D): u, L(D) v)_{0}+(u, v)_{0} .
$$

The completion of $X$ with respect to the scalar product is a Hilbert space $H^{(k)}(G)$.

In [1: Theorem 6] we proved that the domain of the closure $L^{\sim}$ of $L$ in $L^{2}(G)$ coincides with the Hilbert space $H^{(k)}(G), D\left(L^{\sim}\right)=H^{(k)}(G)$. On $H^{(k)}(G)$ we have hence the scalar product $(u, v)_{(k)}=\left(L^{\sim} u, L^{\sim} v\right)_{0}+(u, v)_{0}$. Partial integration in (1.5) yields ${ }^{\prime}$

$$
B(u, \phi)=(L(D) u, \phi)_{0}
$$


for all $u \in X$ and $\phi \in C_{0}{ }^{\infty}(G)$ and since (1.7) depends continuously on $u$ in the topology defined by (1.6) we have further

$$
B(w, \phi)=(L \sim w, \phi)_{0}
$$

for all $w \in H^{(k)}(G)$ and $\phi \in C_{0} \dot{\infty}(G)$.

In [1] we proyed that the elements of $H^{(k)}(G)$ have the same boundary behaviour as the elements of $H_{0} r(G)$, i.e.,

$$
H^{(k)}(G) \cap H_{0} r(G)=H^{(k)}(G)
$$

1.6 In [1] we proved the existence and uniqueness of the solution of a generalized Dirichlet problem. We formulate the results for homogeneous boundary data:

For a given $f \in L^{2}(G)$ there exists a unique element $u \in H_{0} \Gamma(G)$ such that

$$
B(u, \phi)=(f, \phi)_{0}
$$

holds for all $\phi \in C_{0}^{\infty}(G)$.

From the regularity result of [1: Theorem 9] it follows that the solution $u$ of (1.10) lies in $H^{(k)}(G)$.

\section{The resolvent of $L^{\sim}$}

2.1 We first derive a priori estimates for the operator $L^{\sim}$. Take a finite open intervall $J:=\{t \in \mathbf{R} \mid-T<t<T\}$ and let $G$ be a bounded open set in $\mathbf{R}^{n}$ with Property $P$. The set of all functions which obey the condition

$$
\begin{array}{ll}
\omega(x, .) \in C_{0}{ }^{\infty}(J) & \text { for each } x \in G, \\
\omega(., t) \in X & \text { for each } t \in J
\end{array}
$$

, will be denoted by $\mathscr{C}(G \times J)$. On this set we introduce the norm

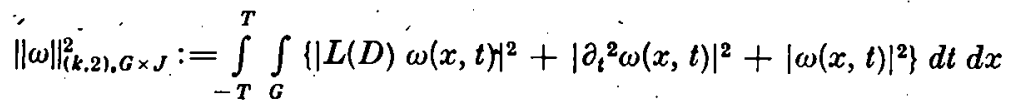

where we use the notation $\partial_{\ell}$ for the differential operator $\partial / \partial t$.

Lemma 1: The estimate

$$
\left\|\left\{L(D)+\mathrm{e}^{i \theta} \partial_{l}^{2}\right\} \omega\right\|_{0, G \times J}^{2} \geqq\|\omega\|_{(k, 2), G \times J}^{2}-\|\omega\|_{0, G \times J}^{2}
$$

holds for each $\theta \in \mathbf{R}$ with $\pi / 2 \leqq \theta \leqq 3 \pi / 2$ and for all $\omega \in \mathscr{C}(G \times J)$.

Proof: For $\omega \in \mathscr{C}(G \times J)$ we have

with

$$
\begin{aligned}
& \left\|\left\{L(D)+\mathrm{e}^{i \theta} \partial_{t}^{2}\right\} \omega\right\|_{0, G \times J}^{2}=\int_{-T}^{T} \int_{G}\left|\left\{L(D)+\mathrm{e}^{i \theta} \partial_{\ell}^{2}\right\} \omega(x, t)\right|^{2} d t d x \\
& =\int_{T}^{T} \int_{G}\left\{|L(D) \omega(x, t)|^{2}+\left|\partial_{t}{ }^{2} \omega(x, t)\right|^{2}\right\} d t d x+\mathrm{e}^{i \theta} \varkappa+\mathrm{e}^{-i \theta} \varkappa \\
& =\|\omega\|_{(k, 2), G \times J}^{2}-\|\omega\|_{0, G \times J}^{2}+2 \operatorname{Re}\left(\mathrm{e}^{i \theta} \varkappa\right)
\end{aligned}
$$

$$
x:=\int_{-T}^{T} \int_{G} \overline{L(D) \omega(x, t)} \partial_{t}{ }^{2} \omega(x, t) d t d x
$$


By partial integration one gets (note that the boundary terms vanish)

$$
\begin{aligned}
& \varkappa_{i}=\int_{-T}^{T} \int_{G} \sum_{\alpha \in T} \overline{D_{x}^{2 a} \omega(x, t)} \cdot \partial_{t}^{2} \omega(x, t) d t d x \\
& =\int_{-T}^{T} \int_{G} \sum_{\alpha \in \Gamma} \overline{D_{x}^{\alpha} \omega(x, t)} D_{x}^{\alpha} \partial_{t}^{2} \omega(x, t) d t d x \\
& \doteq-\int_{-T}^{T} \int_{G} \sum_{a \in \Gamma}\left|D_{x}^{\alpha} \partial_{t} \omega(x, t)\right|^{2} d t d x \text {. }
\end{aligned}
$$

Thus one has $x \in \mathbf{R}, x \leqq 0$. We get therefore by the assumption $2 \operatorname{Re}\left(\mathrm{e}^{\mathrm{i} \theta} x\right)$ $=2 \varkappa \cos \theta \geqq 0$, and (2.2) gives the desired result

Lemma 2: Let $G$ be a bounded open set with Property. $P$. To the operator $L^{\sim}$ there exist two real constants $c^{*}>0$ and $\lambda_{0}>0$ such that ${ }^{1}$ )

$$
c^{*}\left\|\left(L^{\sim}-\lambda I\right) w\right\|_{0 . G} \geqq(1+\cdot \lambda \mid)\|w\|_{0, G}
$$

for all $w \in H^{(k)}(G)$ and for all $\lambda \in \mathbf{C}$ with $|\lambda| \geqq \lambda_{0}$ and $\operatorname{Re} \lambda \leqq 0$.

- Proof: We choose a real valued function $\varrho \in C_{0}{ }^{\infty}(\mathbf{R})$ satisfying the conditions

$$
\varrho(t)=\left\{\begin{array}{lll}
1 & \text { for } & |t| \leqq 1 \\
0 & \text { for } & |t| \geqq 2
\end{array}\right.
$$

and $0 \leqq \varrho(t) \leqq 1$ elsewhere. For an arbitrary function $u \in X$ and an arbitrary $\mu \in \mathbf{R}$ we define the function $\omega$ by

$$
\dot{\omega}(x, t):=u(x) \varrho(t) \mathrm{e}^{i \mu t} .
$$

Now let us take $T>2$. Then one has $\omega \in \mathscr{C}(G \times J)$, and we get by Lemma 1 for $0 \in \mathbf{R}, \pi / 2 \leqq \theta \leqq 3 \pi / 2$,

$$
\left.\cdot\|\omega\|_{(k, 2), G \times J}^{2} . \leqq \| L(D)+\mathrm{e}^{i \theta} \partial_{t}^{2}\right\} \omega\left\|_{0, G \times J}^{2}+\right\| \omega \|_{0, G \times J}^{2} .
$$

We estimate now the first term on the right hand side of (2.3):

$$
\begin{aligned}
& \left\|\left\{L(D)+\mathrm{e}^{i \theta} \partial_{t}{ }^{2}\right\} \omega\right\|_{0, G \times J}^{2} \\
& =\int_{-T}^{T} \int_{G}\left|L(D) \omega(x, t)+\mathrm{e}^{i \theta} u(x) \mathrm{e}^{i \mu t}\left\{\varrho^{\prime \prime}(t)+2 i \mu \varrho^{\prime}(t)-\mu^{2} \varrho(t)\right\}\right|^{2} d t d x \\
& \leqq 2 \int_{-T}^{T} \int_{G}\left\{|\varrho(t)|^{2}\left|L(D) u(x)-\mu^{2} \mathrm{e}^{i \dot{\theta}} u(x)\right|^{2}+|u(x)|^{2}\left|\varrho^{\prime \prime}(t)+2 i \mu \varrho^{\prime}(t)\right|^{2} d t d x\right. \\
& \leqq C_{1} \int_{G}\left|L(D) u(x)-\mu^{2} \mathrm{e}^{i \theta} u(x)\right|^{2} d x+C_{2} \int_{G}|u(x)|^{2} d x \\
& =C_{1}\left\|\left\{L(D)-\mu^{2} \mathrm{e}^{i \theta}\right\} u\right\|_{0, G}^{2}+C_{2}\|u\|_{0, G}^{2}
\end{aligned}
$$

with two positive constants $C_{1}$ and $C_{2}$ (independent of $u$ and $T$ ).

1) By $I$ we denote the identity operator on $L^{2}(G)$. 
The second term on the right hand side of (2.3) satisfies the estinate

$$
\|\omega\|_{0, G \times J^{\prime}}^{2}=\int_{-T}^{T} \int_{G}\left|u(x) \cdot(t) \mathrm{e}^{i \mu t}\right|^{2} d t d x \leqq \bar{C}_{3}\|u\|_{0, G}^{2}
$$

with a third"positive constant $C_{3}$.

By (2.1) we receive for the left hand term of (2.3)

$$
\begin{aligned}
\|\omega\|_{(k, 2), G \times J}^{2} & \geqq \int_{-1}^{1} \int_{G}\left\{|L(D) \omega(x, t)|^{2}+\left|\partial_{t}{ }^{2} \omega(\dot{x}, t)\right|^{2}+|\omega(x, t)|^{2}\right\} d t d x \\
& =2\|L(D) u\|_{0, G}^{2}+2 \mu^{4}\|u\|_{0, G}^{2}+2\|u\|_{0, G}^{2} \\
& \geqq\left(2 \mu^{4}+2\right)\|u\|_{0, G}^{2}
\end{aligned}
$$

From (2.3) we get by the estimates $(2.4)-(2.6)$

$$
\left(2 \mu^{4}+2-C_{2}-C_{3}\right)\|u\|_{0, G}^{2} \leqq C_{1}^{\prime}\left\|\left\{L(D)-\mu^{4} \mathrm{e}^{i \theta}\right\} u\right\|_{0, G}^{2} \cdot
$$

We choose $\lambda_{0}=1+\left(C_{2}+C_{3}\right)^{1 / 2}$. Then for all $\lambda={ }^{-} \mu^{2} \mathrm{e}^{i \theta}$. with $\mu \in \mathbf{R}, \mu^{2} \geqq \lambda_{0}$ and $\pi / 2 \leqq \theta \leqq 3 \pi / 2$ the inequality

$$
c^{*}\|(L(D)-\lambda) u\|_{0, G} \geqq(1+|\lambda|)\|u\|_{0, G}
$$

with $c^{*}:=+C_{1}^{1 / 2}$ is, valid for all $u \in X$. By continuous extension to $H^{(k)}(G)$ we get the assertion of the lemma

\section{- 2.2 Analogously to the élliptic case we prove now}

Lemma 3: Let $G$ be a bounded open set with Property $P$. Then the range of the operator $L \sim-\lambda I: H^{(k)}(G) \rightarrow L^{2}(G)$ coincides with the whole space $L^{2}(G)$ for all $\lambda \in \mathbf{C}$ with $\operatorname{Re} \lambda \leqq 0$, and the operator $L^{\sim}-\lambda I$ is bijective.

Proof: Let be $\lambda \in \mathrm{C}, \operatorname{Re} \lambda \leqq 0$; and take an arbitrary element $f \in L^{2}(G)$. We have to prove that there exists a uniquely determined element $u \cdot \in H^{(k)}(G)$ with $\left(L^{\sim}-\lambda I\right) u=f$.

We define .

$$
B_{\lambda}(u, v):=B(u, v)-\lambda(u, v)_{0, G} \text { for all } u, v \in X
$$

Now one has by (1.3) (ás $\operatorname{Re} \lambda \leqq 0$ ) the estimate

$$
\operatorname{Re} B_{\lambda}(u, u)=B(u, u)-\operatorname{Re} \lambda(u, u)_{0, G} \geqq\|\| u\|\|^{2} \text {. }
$$

On the other hand, the sesquilinear form,$B_{\lambda}(\cdot, \cdot)$ is bounded on $X,\left|B_{\lambda}(u, v)\right|$ $\leqq(1+|\lambda|)\|u\|_{\Gamma}\|v\|_{\Gamma}$. As $B_{\lambda}(\cdot, \cdot)$ can be continuoúly extended to a bounded sesquilinear form with property $(2.7)$ to $H_{0} \Gamma(G)$, we get by the theorem of LAX-MiLaraur (cf. [3: pp. 41-46]) the existence of a unique element $u \in H_{0} r(G)$ such that $(f, \phi)_{0 . G}$ $=B_{\lambda}(u, \phi)$ is valid for all $\phi \in C_{0}^{\infty}(G)$. By the regularity result for the solution $u \in H_{0} r(G)$ of the homogeneous Dirichlet problem mentioned in 1.6 we have $u \in H^{(k)}(G)$.

By partial integration (see (1.5)) we get further

$$
(f, \phi)_{0, G}=B_{\dot{i}}(u, \phi)=\left(\left(\dot{L}^{\sim}-\lambda I\right) . u, \phi\right)_{0}
$$

for all $\phi \in C_{0}^{\infty}(G)$. This proves that the operator $\dot{L}^{\sim}-\lambda I: H^{(k)}(G) \rightarrow L^{2}(G)$ is bi- , jective 
2.3 By Lemma 3 the domain of the resolvent $\left(\lambda I:-L^{\sim}\right)^{-1}$ of the operator $L^{\sim}$ coincides with the whole space $L^{2}(G)$ for all $\lambda \in \mathbf{C}$ with $\operatorname{Re} \lambda \leqq 0$, hence for every such $\lambda$ the operator $\left(\lambda I-L^{\sim}\right)^{-1}$ is bounded. Furthermore we prove the following estimate for the resolvent.

Lem ma 4: Let G be a bounded open set with Property $P$. Then the resolvent $\left(\lambda I-L^{\sim}\right)^{-1}$ of the operator $L^{\sim}$ satisfies the estimate

$$
\left\|\left(\lambda I-L^{\sim}\right)^{-1}\right\| \leqq \frac{c}{1+|\lambda|}
$$

for all $\lambda \in \mathbf{C}$ with $\mathrm{Re} \lambda_{1} \leqq 0$ where $c$ is a positive constant.

Proof: The operator $\left(\lambda I-L^{\sim}\right)^{-1}$ is bounded for every $\lambda \in \mathbf{C}$ with $\operatorname{Re} \lambda \leqq 0$, i.e., for every such $\lambda$ there exists a positive constant $c_{\lambda}$ such that $\left\|\left(\lambda I-L^{\sim}\right)^{-1}\right\| \leqq c_{\lambda}$. Then every $\lambda \in \mathbf{C}$ with $\operatorname{Re} \lambda \leqq 0$ has a neighborhood $U_{\lambda}$ in $\mathbf{C}$ for which

$$
\left\|\left(\lambda^{\prime} I-L^{\sim}\right)^{-1}\right\| \leqq 2 c_{\lambda} \text { for all } \dot{\lambda}^{\prime} \in U_{\lambda} \text {. }
$$

Thus, the resolvent $\left(\lambda I-L^{\sim}\right)^{-1}$ is uniformly bounded on each compact subset of the half plane $\operatorname{Re} \lambda \leqq 0$, and we have with a constant $c_{0}>0$ (which is independent of $\lambda$ )

$$
\left\|\left(\lambda I-L^{\sim}\right)^{-1}\right\| \leqq c_{0}
$$

for all $\lambda \in \mathbf{C}$ with $\operatorname{Re} \lambda \leqq 0$ and $|\lambda| \leqq \lambda_{0}$ (for $\lambda_{0}$ see Lemma 2). On the other hand, by Lemma 2 we have.

$$
\left\|\left(\lambda I-L^{\dot{\tau}}\right)^{-1}\right\| \leqq \frac{c^{*}}{1+|\lambda|}
$$

for all $\lambda \in \mathrm{C}$ with $\operatorname{Re} \lambda \leqq 0$ and $|\lambda| \geqq \lambda_{0}$. Hence with $c:=\max \left\{c^{*}, c_{0}\left(1+\lambda_{0}\right)\right\}$ it follows from (2.9) and (2.10) that the relation

$$
\left\|\left(\lambda I-L^{\sim}\right)^{-1}\right\| \leqq \frac{c}{1+|\lambda|}
$$

holds for all $\lambda \in \mathbf{C}$ with $\operatorname{Re} \lambda \leqq 0$

\section{The Cauchy Problem}

3.1 We will now investigate the generalized Cauchy problem mentioned in the Introduction. Because of (1.9) this problem is an initial-boundary value problem. with generalized homogeneous boundary values on $\partial G$ and with non'-homogeneous initial values on $G$.

Problem $\mathrm{C}$ : Let $G$ be a bounded open set in $\mathbf{R}^{n}$ with Property P. Further let ${ }^{2}$ ) $f: \mathbf{R}_{0}{ }^{+} \rightarrow L^{2}(G)$ be a given uniformly Hölder continuous (with an exponent $\beta, 0<\beta \leqq 1$ ) function on $\mathbf{R}_{0}{ }^{+}$with values in $L^{2}(G), f \in C^{0 . \beta}\left(\mathbf{R}_{0}{ }^{+}, L^{2}(G)\right)$, and $u_{0}$ a given element of $H^{(k)}(G)$. We want to find all functions $i: \mathbf{R}_{0}{ }^{+} \rightarrow H^{(k)}(G)$ from the class $C^{0}\left(\mathbf{R}_{0^{+}}, L^{2}(G)\right)^{\prime}$ $\cap C^{1}\left(\mathbf{R}^{+}, L^{2}(G)\right)$ which solve the generalized evolution equation

$$
\frac{d}{d t} u(t)+L^{\sim} u(t)=f(t) \text { for }{ }^{\prime} t>0
$$

and satisfy the initial condition $u(0)=u_{0}$.

$\left.{ }^{2}\right)$ We use the notations $\mathbf{R}^{+}:=\{r \in \mathbf{R} \mid r>0\}$ and $\mathbf{R}_{0}^{+}:=\mathbf{R}^{+} \cup\{0\}$. 
3.2 We are now able to use for this problem the theory presented by A. Friedian in ' 3 : Part 2, especially 2.1-2.13, pp. 101-158] (cf. also [6: pp. 85-109]). For this theory it is not necessary that $L^{\sim}$ is the closure of an elliptic differential operator but that

$$
D\left(L^{\sim}\right)=H^{(k)}(G)
$$

is dense in $L^{2}(G)$ and that with a constant $c>0$ the estimate

$$
\left\|\left(\lambda I-L^{\sim}\right)^{-1}\right\| \leqq \frac{c}{1 \backslash+|\lambda|}
$$

is valid for all $\lambda \in \mathbf{C}$ with $\operatorname{Re} \lambda \leqq 0$. These conditions guarantee that the operator $-L^{\sim}$ is an infinitesimal generator of an analytic semigroup of bounded linear operators in $L^{2}(G)$, with the help of this fact: one proves the existence and uniqueness of the fundamental solution $V(\cdot, \tau)$ for the operator $\frac{d}{d t}+L^{\sim}$.

By a fundamental solution we mean a function ${ }^{3}$ )

$$
V(\cdot, \cdot):\left\{t \in \mathbf{R}\{\tau \leqq t<\infty\} \times\{\tau \in \mathbf{R} \mid \tau \geqq 0\} \rightarrow \mathscr{B}\left(L^{2}(G)\right)\right.
$$

with the properties:

I. The operator $V(t, \tau)\left(\epsilon \mathscr{B}\left(L^{2}(G)\right)\right)$ is strongly continuous in $t, \tau$ for $0 \leqq \tau \leqq t<\infty$.

II. The derivative $\frac{\partial}{\partial t} V(t, \tau)$ exists in the strong topology of $\mathscr{B}\left(L^{2}(G)\right)$ and belongs to $\mathscr{B}\left(L^{2}(G)\right)$ for $0 \leqq \tau<t<\infty$ and is also strongly continuous in $t$ for $\tau<t<\infty$.

III: The range of $V(t, \tau)$ lies in $D\left(L^{\sim}\right)\left(=H^{(k)}(G)\right)$ for all $t, \tau$ with $0^{-} \leqq \tau<t<\infty$.

IV. The function $V(\cdot, \tau)$ is the solution of the Cauchy problem

$$
\frac{\partial}{\partial t} V(t, \tau)+L^{\sim} V(t, \tau)=0 \text { for } \tau<t<\infty
$$

and $V(\tau, \tau)=I$.

Finally we get from the considerations of A. Friedman [3: p. 109].

Theorem 5: Problem $C$ has a unique solution $u$. This solution has the expression

$$
u(t):=\dot{V}(t, 0) u_{0}+\int_{0}^{t} V(t, s) \cdot f(s) d s .
$$

The authors would like to thank I. S. LouhrvaARA for his comments while writing this paper.

\section{REFERENCES}

[1] Doppez, K., and N. JACOB: A non-hypoelliptic Dirichlet problem from stochastics. Ann. Acad. Sci. Fenn. Ser. A. I. Math. 8 (1983), 375-389.

[2] Dynkin, E. B.: Harmonic functions associated with several Markov processes. Adv. in Appl. Math. 2 (1981), 260-283.

[3] Frifdman, A.: Partial differential equations. New York-Chicago-etc. 1969.

[4] Hudebrandt, S.: Lineare elliptische Differentialgleichungen. (Vorlesung, Mainz 1966,

${ }^{3}$ ) By $\mathscr{B}\left(L^{2}(G)\right)$ we denote the Banach space of all bounded linear operators in $L^{2}(G)$ equipped with the operator norm. 
als Manuskript vervielfältigt.) Johañnes-Gutenberg-Universität, Mathematisches Institut. Mainz 1966.

[5] JАсов, N.: Oǹ generalized Dirichlet problems. Math. Scand. (to appear).

[6] Showalter, R. E.: Hilbert space methods for partial differential equations. London-San Francisco-Melbourne 1977.

Manuskripteingang: 15.08.1983

\section{VERFASSER:}

Prof. Dr. KARL Doppel and Dr. Niels JACOB

Fachbereich Mathematik der Freien Universität Berlin-West 33; Arnimallee 2-6 